\title{
Sintomatología ansiosa-depresiva en relación con el funcionamiento familiar en un grupo de adolescentes de Lima Norte
}

\author{
Anxious-depressive symptoms in relation to family functioning in a group of \\ adolescents from North Lima
}

Tafur-Orahulio, Yolanda Milena ${ }^{1}$

\begin{abstract}
Resumen
Objetivo: determinar la relación entre la percepción del funcionamiento familiar y la sintomatología ansiosa-depresiva en alumnos de secundaria de un colegio público en Lima Norte. Materiales y métodos: el tipo de estudio fue correlacional y el diseño de la investigación fue de corte transversal. La muestra estuvo conformada por 190 alumnos de secundaria de un colegio del distrito de Independencia. A nivel inferencial, se empleó la prueba de Chi cuadrado con un nivel de significancia menor e igual a 0.05 . Resultados: el $67 \%$ y el $51 \%$ de la muestra evidencia presencia de sintomatología ansiosa y depresiva respectivamente; así mismo el 32\% de adolescentes tienen una percepción familiar disfuncional de tipo leve. De igual forma, se encontró relación entre la percepción del funcionamiento familiar y la sintomatología ansiosa $(\mathrm{p}=0.006)$ y depresiva $(\mathrm{p}=0.000)$. Por otro lado, se evidenció relación entre la percepción del funcionamiento familiar y el rendimiento académico $(\mathrm{p}=0.000)$ con el tipo de familia $(\mathrm{p}=0.001)$ y con la cantidad de hermanos $(\mathrm{p}=0.033)$. Conclusión: los estudiantes que reportaron disfunción familiar presentan sintomatología ansiosa-depresiva. Puesto que la mayoría de los estudiantes percibe sus núcleos parentales con inatención y escaso compromiso en su desarrollo personal reflejando su insatisfacción a través de signos de ansiedad o depresión. Asimismo, los factores que favorecen esta percepción de disfunción son el tipo de familia, la cantidad de hermanos y la impresión de un rendimiento académico regular.
\end{abstract}

Palabras clave: Ansiedad; Relaciones Familiares; Adolescente; Tristeza; Relaciones Interpersonales (Fuente: DeCS).

\begin{abstract}
Objective: to determine the relationship between the perception of family functioning and anxiety-depressive symptoms in secondary school students from a public school in North Lima. Materials and methods: the type of study used was correlational; likewise, the research design was cross-sectional. The sample consisted of 190 3rd, 4th and 5th grade secondary students from a school in the Independencia district and was selected through a non-probability sample. At the inferential level, the Chi-square statistical test was used with a significance level less than and equal to 0.05; while at a descriptive level frequencies and percentages were used. Results: $67 \%$ and $51 \%$ of the representative sample show presence of anxious and depressive symptoms, respectively; Likewise, 32\% of adolescents have a mild dysfunctional family perception. Similarly, a relationship was found between the perception of family functioning and anxious $(p=0.006)$ and depressive $(\mathrm{p}=0.000)$ symptoms. On the other hand, there was a relationship between the perception of family functioning and academic performance $(\mathrm{p}=0.000)$ with the type of family $(\mathrm{p}=0.001)$ and with the number of siblings $(\mathrm{p}=0.033)$. Conclusion: students who reported family dysfunction present anxious-depressive symptoms. Since many students perceive their parental nuclei with inattention and little commitment in their personal development, reflecting their dissatisfaction through signs of anxiety or depression. Likewise, the factors that favor having this perception of dysfunction are the type of family, the number of siblings and the impression of a regular academic performance.
\end{abstract}

Keys words: Anxiety; Family relationships; Teen; Sadness; Interpersonal Relations (Source: DeCS).

Para citar:

Tafur YM. Sintomatología ansiosa-depresiva en relación con el funcionamiento familiar en un grupo de adolescentes de Lima Norte. CASUS. 2020;5(1):18-25.

DOI: $10.35626 /$ casus. 1.2020 .228

${ }^{1}$ Colegio Pamer-Magdalena-Perú.

Correo electrónico: milenaorahulio@gmail.com
Fecha de recepción: 11-12-20

Fecha de envío a pares: 16-12-19

Fecha de aprobación por pares: 02-04-20

Fecha de aceptación: 05-04-20 


\section{INTRODUCCIÓN}

A nivel internacional, la sintomatología ansiosadepresiva se evidencia como uno de los problemas de salud mental más frecuentes, y pueden afectar severamente la calidad de vida de la persona. Las estimaciones de prevalencia mundial refieren que un $4.4 \%$ de la población padece de trastorno depresivo y un $3.6 \%$, trastorno de ansiedad. Específicamente, la depresión es más común en las mujeres $(5.1 \%)$ que en los hombres $(3,6 \%)$ y la prevalencia en cuanto a la ansiedad predomina en las mujeres con un $4.6 \%$ a diferencia de los hombres con un 2.6\% (1). En América del Sur, se estima un $15 \%$ de prevalencia respecto al trastorno depresivo y un $7.7 \%$ de la población femenina sufre trastornos de ansiedad respecto a los varones (3.6\%) (1).

En el Perú, estudios del Instituto Nacional de Salud Mental "Honorio Delgado Hideyo Noguchi" indican que la sintomatología ansiosa-depresiva se encuentra entre el $10 \%$ y $15 \%$ en la escala de trastornos mentales más comunes en el país (2). Los trastornos ansiosos-depresivos en adolescentes son considerados trastornos internalizantes, en la medida que afectan principalmente el ámbito de las emociones y las relaciones interpersonales (3). Por ello, el estudio de las manifestaciones depresivas en la adolescencia temprana es particularmente relevante, pues en este período vital se da un incremento notorio de estas dificultades respecto a los períodos previos de la vida, con impactos múltiples en lo personal y en su entorno $(4,5)$.

Es así como la sintomatología ansiosa-depresiva ha sido estudiada junto a la percepción del funcionamiento familiar considerándolos factores que se relacionan en la etapa de la adolescencia, viendo que estos dos componentes interfieren de manera activa en la personalidad de los adolescentes que se encuentran en ambientes familiares con disfunción (6). La familia es el grupo primario de pertenencia de todas las personas, unido entre sí por vínculos de consanguinidad, filiación biológica o adoptiva y de alianza (7). En este sentido, se destaca la relevancia de profundizar sobre el apoyo y la funcionalidad familiar en el campo de la salud. Según Smilkstein, la funcionalidad familiar es definida como la percepción del cuidado y apoyo que un individuo recibe de su familia (7). El sistema familiar catalogado como fuente de ayuda emocional durante la adolescencia muestra que la percepción de un contexto familiar cohesivo es una condición importante para el desarrollo óptimo de los miembros de la familia $(6,7)$.

Puesto que, el funcionamiento familiar es la capacidad que tiene la familia de satisfacer las necesidades de sus miembros y adaptarse a las situaciones de cambio. Se considera funcional cuando es capaz de propiciar la solución a los problemas, de modo que estos no lleguen a afectar a la satisfacción de las necesidades de sus miembros. Para ello debe mantener su organización, desarrollar procesos familiares y realizar las actividades de la vida diaria manteniendo un futuro seguro (8).

Debido a esto, el desarrollo de estos síntomas ansioso-depresivos se relaciona con una percepción deficiente del funcionamiento familiar y esto sucede cuando los adolescentes no sienten un clima de apoyo y seguridad, por lo que ellos estarán propensos a manifestar dichos síntomas que dificultan su desenvolvimiento cotidiano (9). Las conductas de riesgo y problemas emocionales son más frecuentes en adolescentes provenientes de familias percibidas como disfuncionales, manifestándose como un factor de riesgo (8).

En la actualidad, la información encontrada acerca de la relación entre estas dos variables no abarca la población peruana adolescente. Por ello, la realización de estudios sobre este grupo sería relevante, de modo que se pueda aportar al conocimiento y prevención sobre la sintomatología ansiosa-depresiva. Finalmente, con lo expuesto anteriormente, se planteó como objetivo determinar la relación entre la sintomatología ansiosadepresiva y la percepción del funcionamiento familiar en alumnos de secundaria de un colegio público en Lima Norte.

\section{MATERIALES Y MÉTODOS}

El estudio fue de tipo correlacional de corte transversal. La muestra estuvo conformada por 190 
alumnos de un colegio de Lima Norte seleccionados por un muestreo no probabilístico. Las variables principales fueron: sintomatología ansiosadepresiva y percepción del funcionamiento familiar.

Se define por sintomatología ansiosa-depresiva a la expresión de actitudes de dos trastornos emocionales complejos que implican problemas en el pensamiento y conductas que pueden llegar a afectar severamente la calidad de vida de las personas (10). Esta variable fue medida a través de la Escala de Ansiedad y Depresión de Goldberg, la cual fue adaptada por Osada y colaboradores en el año 2010 (10). Es de naturaleza categórica dicotómica y considera las dimensiones de ansiedad (>2) y depresión (>4) y se categorizó en sí y no.

La percepción del funcionamiento familiar se define como el grado de satisfacción de la persona dentro del ámbito familiar y la forma en que esta percepción se manifiesta en las relaciones interpersonales (11). Se midió esta variable con el instrumento del APGAR- familiar de Smilkstein, el mismo que fue adaptado en Perú por Castilla y colaboradores en el año 2014 (12). Es de naturaleza categórica politómica ordinal y está agrupada en cuatro niveles: buena función familiar (18-20), disfunción familiar leve (14-17), disfunción familiar moderada (10-13) y disfunción familiar severa (9-0).

Las variables sociodemográficas de naturaleza cualitativa fueron recolectadas a través de un ficha de datos sociodemográficos que consideró: sexo, dicotómica (femenino, masculino); edad, dicotómica (14 - 15 y 16 - 17 años); año de estudio, politómica ordinal (3ero. secundaria, 4to. secundaria y 5to. secundaria); cantidad de hermanos, politómica ordinal (1-2, 3-4 y 5-6 hermanos); tipo de familia, politómica nominal (nuclear, extensa, monoparental, ensamblada); aporte económico en casa, politómica nominal (mamá, papá o hermanos); distrito de procedencia, politómica nominal (Independencia, Los Olivos, San Martin de Porres y Rímac), percepción del rendimiento académico, politómica ordinal (bueno, regular, malo).
Tabla 1. Descripción de la muestra de adolescentes

\begin{tabular}{|c|c|c|}
\hline & $\mathbf{n}$ & $\%$ \\
\hline \multicolumn{3}{|l|}{ Sexo } \\
\hline Femenino & 96 & 50.53 \\
\hline Masculino & 94 & 49.47 \\
\hline \multicolumn{3}{|l|}{ Edad } \\
\hline $14-15$ & 132 & 69.47 \\
\hline $16-17$ & 58 & 30.53 \\
\hline \multicolumn{3}{|l|}{ Año de estudio } \\
\hline 3ero. & 76 & 40.00 \\
\hline 4to. & 80 & 42.11 \\
\hline 5to. & 34 & 17.89 \\
\hline \multicolumn{3}{|l|}{ Cantidad de hermanos } \\
\hline $1-2$ & 105 & 55.56 \\
\hline $3-4$ & 58 & 30.69 \\
\hline $5-6$ & 26 & 13.76 \\
\hline \multicolumn{3}{|l|}{ Distrito de procedencia } \\
\hline Independencia & 183 & 96.32 \\
\hline Los Olivos & 3 & 1.58 \\
\hline San Martin de Porres & 2 & 01.05 \\
\hline Rímac & 2 & 01.05 \\
\hline \multicolumn{3}{|l|}{ Tipo de familia } \\
\hline Nuclear & 102 & 53.68 \\
\hline Extensa & 11 & 5.79 \\
\hline Monoparental & 77 & 40.53 \\
\hline \multicolumn{3}{|l|}{ Aporte económico en casa } \\
\hline Mamá & 67 & 35.26 \\
\hline Papá & 117 & 61.58 \\
\hline Hermanos & 6 & 3.16 \\
\hline \multicolumn{3}{|l|}{ Rendimiento académico } \\
\hline Bueno & 4 & 2.11 \\
\hline Regular & 129 & 67.89 \\
\hline Malo & 57 & 30.00 \\
\hline \multicolumn{3}{|l|}{ Sintomatología ansiosa } \\
\hline Sí & 128 & 67.37 \\
\hline No & 62 & 32.63 \\
\hline \multicolumn{3}{|l|}{ Sintomatología depresiva } \\
\hline Sí & 97 & 51.05 \\
\hline No & 93 & 48.95 \\
\hline \multicolumn{3}{|l|}{ Percepción del funcionamiento familiar } \\
\hline Buena función familiar & 26 & 13.68 \\
\hline Disfunción familiar leve & 68 & 35.79 \\
\hline Disfunción familiar moderada & 60 & 31.58 \\
\hline Disfunción familiar severa & 36 & 18.95 \\
\hline
\end{tabular}

Para el análisis estadístico se utilizó el software de Stata versión 14. Para el análisis descriptivo se determinaron frecuencias y porcentajes en todas las variables categóricas. A nivel inferencial, para hallar la relación entre las variables principales se empleó Chi-cuadrado con un nivel de significancia de 0.05. Esta investigación fue aprobada por el Comité de Ética de la Universidad Católica Sedes Sapientiae y la información de los participantes fue 
Tabla 2. Asociación entre la sintomatología ansiosa - depresiva, percepción del funcionamiento familiar y variables sociodemográficas

\begin{tabular}{|c|c|c|c|c|c|c|}
\hline & \multicolumn{3}{|c|}{ Sintomatología ansiosa } & \multicolumn{3}{|c|}{ Sintomatología depresiva } \\
\hline & Sí & No & & Sí & No & \\
\hline & n $(\%)$ & n $(\%)$ & $\mathbf{p}$ & n $(\%)$ & n $(\%)$ & p \\
\hline Percepción del funcionamiento familiar & & & 0.006 & & & 0.000 \\
\hline Buena función familiar & $16(61.54)$ & $10(38.46)$ & & $8(30.77)$ & $18(69.23)$ & \\
\hline Disfunción familiar leve & $40(58.82)$ & $28(41.18)$ & & $28(41.18)$ & $40(58.82)$ & \\
\hline Disfunción familiar moderada & $39(65.00)$ & $21(35.00)$ & & $32(53.33)$ & $28(46.67)$ & \\
\hline Disfunción familiar severa & $33(91.67)$ & $3(8.33)$ & & $29(80.56)$ & $7(19.44)$ & \\
\hline Sexo & & & 0.099 & & & 0.042 \\
\hline Femenino & $70(72.92)$ & $26(27.08)$ & & $56(58.33)$ & $40(41.67)$ & \\
\hline Masculino & $58(61.70)$ & $36(38.30)$ & & $41(43.62)$ & $53(56.38)$ & \\
\hline Edad & & & 0.756 & & & 0.411 \\
\hline $14-15$ & $88(66.67)$ & $44(33.33)$ & & $70(53.03)$ & $62(46.97)$ & \\
\hline $16-17$ & $40(68.97)$ & $18(31.03)$ & & $27(46.55)$ & $31(53.45)$ & \\
\hline Año de estudio & & & 0.576 & & & 0.416 \\
\hline 3ero. & $52(68.42)$ & $24(31.58)$ & & $43(56.58)$ & $33(43.42)$ & \\
\hline 4to. & $51(63.75)$ & $29(36.25)$ & & $39(48.75)$ & $41(51.25)$ & \\
\hline 5to. & $25(73.53)$ & $9(26.47)$ & & $15(44.12)$ & $19(55.88)$ & \\
\hline Cantidad de hermanos & & & 0.3445 & & & 0.122 \\
\hline $1-2$ & $75(71.43)$ & $30(28.57)$ & & $58(55.24)$ & $47(44.76)$ & \\
\hline $3-4$ & $35(60.34)$ & $23(39.66)$ & & $23(39.66)$ & $35(60.34)$ & \\
\hline $5-6$ & $17(65.38)$ & $9(34.62)$ & & $15(57.69)$ & $11(42.31)$ & \\
\hline Distrito de procedencia & & & 0.572 & & & 0.364 \\
\hline Independencia & $123(67.21)$ & $60(32.79)$ & & $95(51.91)$ & $88(48.09)$ & \\
\hline Los Olivos & $3(100.0)$ & $0(0.00)$ & & $0(0.00)$ & $3(100.0)$ & \\
\hline San Martin de Porres & $1(50.00)$ & $1(50.00)$ & & $1(50.00)$ & $1(50.00)$ & \\
\hline Rímac & $1(50.00)$ & $1(50.00$ & & $1(50.00)$ & $1(50.00)$ & \\
\hline Tipo de familia & & & 0.271 & & & 0.204 \\
\hline Nuclear & $64(62.75)$ & $38(37.25)$ & & $46(45.10)$ & $56(54.90)$ & \\
\hline Extensa & $7(63.64)$ & $4(36.36)$ & & $6(54.55)$ & $5(45.45)$ & \\
\hline Monoparental & $57(74.03)$ & $20(25.97)$ & & $45(58.44)$ & $32(41.56)$ & \\
\hline Aporte económico en casa & & & 0.156 & & & \\
\hline Mamá & $47(70.15)$ & $20(29.85)$ & & $37(55.22)$ & $30(44.78)$ & 0.155 \\
\hline Papá & $75(64.10)$ & $42(35.90)$ & & $55(47.01)$ & $62(52.99)$ & \\
\hline Hermanos & $6(100.0)$ & $0(0.00)$ & & $5(83.33)$ & $1(16.67)$ & \\
\hline Rendimiento académico & & & 0.295 & & & 0.060 \\
\hline Bueno & $36(63.19)$ & $21(36.84)$ & & $22(38.60)$ & $35(61.40)$ & \\
\hline Regular & $88(68.22)$ & $41(31.78)$ & & $72(55.81)$ & $57(44.19)$ & \\
\hline Malo & $4(100.0)$ & $0(0.00)$ & & $3(75.00)$ & $1(25.00)$ & \\
\hline
\end{tabular}

protegida de acuerdo con los principios éticos en investigación con seres humanos.

\section{RESULTADOS}

En la tabla 1, se reflejó que, de los 190 alumnos encuestados, el $50 \%$ fue de sexo femenino, el $69 \%$ reportó edades entre 14 y 15 años, el $42 \%$ cursaba el 4to año de secundaria, el $55 \%$ tuvo entre 1 a 2

hermanos, el $96 \%$ residía en el distrito de Independencia y el $54 \%$ provenía de una familia nuclear al momento de la encuesta. En cuanto al aporte económico, en casa el $61 \%$ tenía como encargado al padre y el $68 \%$ percibían su rendimiento académico como regular. Entre los estudiantes se observó el padecimiento de sintomatología ansiosa en un $67 \%$ y de sintomatología depresiva en un $51 \%$. Mientras que se reflejó que entre los estudiantes encuestados un $36 \%$ percibían su funcionamiento familiar como una disfunción familiar de tipo leve. En la tabla 2, se demostró que existe relación 
Tabla 3. Asociación entre la percepción del funcionamiento familiar y las variables sociodemográficas

\begin{tabular}{|c|c|c|c|c|c|c|}
\hline & \multicolumn{5}{|c|}{ Percepción del funcionamiento familiar } \\
\hline & & $\begin{array}{c}\text { Disfunción } \\
\text { familiar } \\
\text { severa } \\
\text { n }(\%) \\
\end{array}$ & $\begin{array}{c}\text { Disfunción } \\
\text { familiar } \\
\text { moderada } \\
\text { n }(\%) \\
\end{array}$ & $\begin{array}{c}\text { Disfunción } \\
\text { familiar } \\
\text { leve } \\
\text { n }(\%) \\
\end{array}$ & $\begin{array}{c}\text { Buena } \\
\text { función } \\
\text { familiar } \\
\text { n }(\%)\end{array}$ & $\mathbf{p}$ \\
\hline \multirow[t]{3}{*}{ Sexo } & & & & & & 0.729 \\
\hline & Femenino & $19(19.79)$ & $29(30.21)$ & $37(38.54)$ & $11(11.46)$ & \\
\hline & Masculino & $17(18.09)$ & $31(32.98)$ & $31(32.98)$ & $15(15.96)$ & \\
\hline \multirow[t]{3}{*}{ Edad } & & & & & & 0.365 \\
\hline & $14-15$ & $23(17.42)$ & $44(33.33)$ & $50(37.88)$ & $15(11.36)$ & \\
\hline & $16-17$ & $13(22.41)$ & $16(27.59)$ & $18(31.03)$ & $11(18.97)$ & \\
\hline \multicolumn{2}{|c|}{ Año de estudio } & & & & & 0.212 \\
\hline & 3ero. & $9(11.84)$ & $26(34.21)$ & $34(44.74)$ & $7(9.21)$ & \\
\hline & 4to. & $19(23.75)$ & $24(30.00)$ & $24(30.00)$ & $13(16.25)$ & \\
\hline & 5to. & $8(23.53)$ & $10(29.41)$ & $10(29.41)$ & $6(17.65)$ & \\
\hline \multicolumn{3}{|c|}{ Cantidad de hermanos } & & & & 0.033 \\
\hline & $1-2$ & $27(25.71)$ & $13(50.00)$ & $5(19.23)$ & $5(19.23)$ & \\
\hline & $3-4$ & $6(10.34)$ & $20(34.48)$ & $22(37.93)$ & $10(17.24)$ & \\
\hline & $5-6$ & $3(11.54)$ & $27(25.71)$ & $40(38.10)$ & $11(10.48)$ & \\
\hline \multicolumn{3}{|c|}{ Distrito de procedencia } & & & & 0.419 \\
\hline & Independencia & $35(19.13)$ & $59(32.24)$ & $65(35.52)$ & $24(13.11)$ & \\
\hline & Los Olivos & $0(0.00)$ & $1(33.33)$ & $2(66.67)$ & $0(0.00)$ & \\
\hline & San Martin de Porres & $1(50.00)$ & $0(0.00)$ & $0(0.00)$ & $1(50.00)$ & \\
\hline & Rímac & $0(0.00)$ & $0(0.00)$ & $1(50.00)$ & $1(50.00)$ & \\
\hline \multicolumn{3}{|c|}{ Tipo de familia } & & & & 0.001 \\
\hline & Nuclear & $16(15.69)$ & $21(20.59)$ & $44(43.14)$ & $21(20.59)$ & \\
\hline & Extensa & $4(36.36)$ & $3(27.27)$ & $4(36.36)$ & $0(0.00)$ & \\
\hline & Monoparental & $16(20.78)$ & $36(46.75)$ & $20(25.97)$ & $5(6.49)$ & \\
\hline \multicolumn{3}{|c|}{ Aporte económico en casa } & & & & 0.109 \\
\hline & Mamá & $17(25.37)$ & $26(38.81)$ & $18(26.87)$ & $6(8.96)$ & \\
\hline & Papá & $17(14.53)$ & $32(27.35)$ & $48(41.03)$ & 20(17.09) & \\
\hline & Hermanos & $2(33.33)$ & $2(33.33)$ & $2(33.33)$ & $0(0.00)$ & \\
\hline \multicolumn{3}{|c|}{ Rendimiento académico } & & & & 0.000 \\
\hline & Bueno & $2(3.51)$ & $20(35.09)$ & $18(31.58)$ & $17(29.82)$ & \\
\hline & Regular & $32(24.81)$ & $38(29.46)$ & $50(38.76)$ & $9(6.98)$ & \\
\hline & Malo & $2(50.00)$ & $2(50.00)$ & $0(0.00)$ & $0(0.00)$ & \\
\hline
\end{tabular}

significativa entre la percepción del funcionamiento familiar y la sintomatología ansiosa-depresiva: ansiedad $\quad(\mathrm{p}=0.006)$ y depresión $\quad(\mathrm{p}=0.000)$. Mostrando que el 59\% y $65 \%$ de los estudiantes que reportaron una percepción familiar disfuncional de tipo leve y moderada respectivamente, presentaron sintomatología ansiosa, y el 53\% de la población que reportó una percepción familiar disfuncional de tipo moderado padece de sintomatología depresiva.

En cuanto a las variables sociodemográficas, se encontró relación significativa entre el sexo y la sintomatología depresiva $(\mathrm{p}=0.042)$, mostrando que el $58 \%$ de las estudiantes presentaron síntomas de depresión. El resto de las variables sociodemográficas no mostró resultados con significancia (ver tabla 2).

En la tabla 3, se manifestó relación significativa entre la cantidad de hermanos y la percepción del funcionamiento familiar ( $\mathrm{p}=0.033$ ), mostrando que un $38 \%$ y $26 \%$ de estudiantes que tiene de 5 a 6 hermanos presentaron una percepción familiar disfuncional de tipo leve y moderada respectivamente. Del mismo modo, se evidenció relación entre el tipo de familia $(\mathrm{p}=0.001)$ y la percepción del funcionamiento familiar, reflejando que el $47 \%$ de estudiantes con familias monoparentales reportaron una percepción familiar disfuncional de tipo moderado. Asimismo, se encontró relación significativa entre el rendimiento 
académico y la percepción del funcionamiento familiar $(p=0.00)$ reportando que un $39 \%$ de alumnos con un rendimiento académico regular tienen una percepción familiar disfuncional de tipo leve. El resto de las variables sociodemográficas no mostró resultados significativos (ver tabla 3).

\section{DISCUSIÓN}

Los resultados demuestran que existe relación significativa entre la percepción del funcionamiento familiar y la sintomatología ansiosa-depresiva. Además, se observó que la sintomatología depresiva se relacionó con el sexo. Del mismo modo, se evidenció que la percepción del funcionamiento familiar se relacionó con el tipo de familia, la cantidad de hermanos y el rendimiento académico.

Los alumnos que reportaron la presencia de una percepción familiar disfuncional de tipo leve y moderado evidenciaron sintomatología ansiosa. Otros estudios con resultados semejantes señalan que la disfuncionalidad familiar es un factor asociado a la ansiedad $(13,14)$. Esto podría explicarse afirmando la importancia que tiene la familia como factor protector de las emociones de sus miembros (15). Ya que las familias con disfunción familiar presentan una cohesión alta y una adaptación baja, haciendo que los miembros perciban a la familia como una estructura rígida, con una comunicación pobre, sin posibilidades de cambio o afecto y una interacción que impide el crecimiento personal sintiéndose aislados, sin vínculos emocionales y haciéndose más propensos a padecer de trastornos internalizantes (ansiedad y depresión) (16).

Por otro lado, los alumnos que manifestaron una percepción familiar disfuncional de tipo moderado evidencian sintomatología depresiva. Estos resultados coinciden con otra investigación reflejando que los alumnos con una familia monoparental disfuncional cuentan con problemas para la resolución de los conflictos y la canalización de las emociones (17). Puesto que, en un ambiente disfuncional los adolescentes se encontrarán propensos a presentar trastornos de conducta, depresión o intentos suicidas en respuesta a su inestabilidad emocional (17). Asimismo, los adolescentes del presente estudio reportaron que en su institución educativa no existe un departamento de psicología que realice actividades de prevención y promoción de la salud mental. Más aún, cuando se solicita la participación de los padres de familia en las actividades académicas de sus hijos, estos no asisten demostrando falta de compromiso y cuidado hacia aquellos.

Así mismo, los estudiantes de sexo femenino denotan con mayor frecuencia sintomatología depresiva. Un estudio realizado en instituciones educativas públicas de Colombia evidenció resultados similares, señalando que la mayor frecuencia de depresión se encuentra en el sexo femenino en comparación al sexo masculino (18). Según la psicología cognitiva, la depresión se manifiesta a nivel de pensamiento (pensamientos automáticos negativos) y afecto (afectos negativos). En efecto, otro estudio comprobó que las mujeres adolescentes presentan puntajes más bajos en pensamientos positivos y puntajes mayores en pensamientos automáticos negativos, orientación negativa hacia el problema y necesidad de aprobación y éxito; haciendo que se generen en ellas de manera más rápida los afectos negativos de tristeza y ansiedad (19). Finalmente, a pesar de que la depresión es considerada la principal causa mundial de discapacidad según los porcentajes de morbilidad, la diferencia de la prevalencia según el sexo es cada vez más corta (20).

Además, los alumnos con una cantidad de cinco a seis hermanos presentaron una disfunción familiar de tipo leve y moderado. Por el contrario, se encontró que a mayor cantidad de hermanos dentro de una familia existe una mejor adaptación y cohesión por lo que contarían con buena percepción del funcionamiento familiar (21). Este resultado de la investigación se puede explicar por la propia característica familiar de la población, ya que estas se encuentran dentro de una estructura de parentesco monoparental. De manera que, una figura parental ausente genera que la otra sea la única responsable del cuidado de los hijos, y que si estos son mayores en cantidad quizás no podrán tener una adecuada atención, insatisfacción de sus 
necesidades básicas en cuanto al afecto y la atención necesaria dentro de este contexto familiar.

Del mismo modo, los alumnos con familias monoparentales reportaron una percepción familiar disfuncional de tipo moderado. Otro estudio encontró resultados similares, donde señalan que las familias monoparentales cuentan con una disfuncionalidad familiar moderada en su cohesión, límites y adaptabilidad inadecuados (22). Esto podría explicarse porque en estas familias no existe una persona que ejerza el liderazgo, los roles son más o menos estables, las decisiones no son consensuadas, sino más bien son llevadas a cabo impulsivamente, no existen normas y las reglas cambian constantemente (22). Asimismo, evidencian conductas intrusivas, un elevado apego emocional del progenitor presente hacia sus hijos dificultando la autonomía e independencia de estos y haciendo que se genere un grado de satisfacción familiar bajo (22).

Finalmente, los alumnos que se perciben con un rendimiento académico regular poseen una disfuncionalidad familiar de tipo leve. Resultados similares se encontraron en otro estudio evidenciando relación entre los comportamientos de los miembros de una familia disfuncional con el desempeño académico de los menores hijos de esta (23). Al respecto, según el modelo de aprendizaje, se considera a la familia dentro del proceso de autorregulación de los miembros. En las familias disfuncionales esta autorregulación se encuentra muy limitada, ya que no existe un adecuado modelo, apoyo motivacional, ayuda y recompensas haciendo que el menor no sienta la motivación de desempeñarse académicamente de forma adecuada (23).

Entre las limitaciones del estudio se puede considerar la presencia de la deseabilidad social por parte de los alumnos influyendo en la respuesta de los instrumentos, hallando resultados incongruentes al querer dar una buena imagen personal. Así mismo, el instrumento utilizado para medir el funcionamiento familiar no consideró las dimensiones de cohesión, adaptabilidad mostrando solamente el nivel de funcionamiento familiar que tenía el estudiante en su hogar. En cuanto a las fortalezas se encuentra el uso de instrumentos estandarizados para medir el fenómeno.

\section{CONCLUSIONES}

La sintomatología ansiosa-depresiva se relaciona con la percepción del funcionamiento familiar. Puesto que la mayoría de los estudiantes percibe sus núcleos parentales con inatención y escaso compromiso en su desarrollo personal reflejando su insatisfacción a través de signos de ansiedad o depresión. Asimismo, los factores que favorecen esta percepción de disfunción son el tipo de familia, la cantidad de hermanos y la impresión de un rendimiento académico regular. De igual forma, las características con las que cuenta la población: el tipo de familia del que proceden, la cantidad de hermanos y el rendimiento académico favorecen que la percepción del funcionamiento familiar sea baja. Puesto que no se pueden cubrir completamente las necesidades básicas como afecto y cuidado de manera parcial a todos los miembros de la familia repercutiendo en el desempeño escolar de estos adolescentes.

Con los hallazgos encontrados se busca fomentar la elaboración de planes de evaluación e intervención sistémicos a nivel personal, familiar y social, para mejorar los lazos afectivos entre los adolescentes y sus familias. Esto, mediante el desarrollo programas formativos sobre el desarrollo y la potencialización del funcionamiento familiar para los adolescentes, fortaleciendo el marco de desarrollo positivo adolescente. 


\section{REFERENCIAS BIBLIOGRÁFICAS}

1. Organización Panamericana de la Salud. Depresión y otros trastornos mentales comunes. Estimaciones sanitarias mundiales [internet]. Washington, D.C.: OPS; 2017. 2017.

2. Instituto Especializado de Salud Mental. Estudio Epidemiológico Metropolitano en Salud Mental 2002. Informe General. Anales de Salud Mental. 2002;18(1-2):120125.

3. Garcia R. Trastornos ansiosos y depresivos en adolescentes. Revista Medica Clinica Condes. 2011;22(1):77-84.

4. Harrington R: Affective disorders. Child and Adolescent Psychiatry. Oxford: Blackwell Science;2002.

5. Zamora M. Sintomatología ansiosa-depresiva en una muestra infantil y su relación con el estilo educativo percibido [Tesis de pregrado]. Andalucía: Universidad de Jaén; 2016.

6. Luna C. Funcionamiento familiar conflictos con los padres y satisfacción con la vida de familia en adolescentes bachilleres. Acta Colombiana de Psicología. 2012;15(1):77-85.

7. Retamales J, Behn V, Merino J. Funcionamiento familiar de trabajadores - Jefes de hogar de turnos de una empresa de Talcahuano en Chile 2003. Revista de Ciencia y Enfermedad. 2004;10(1):23-30.

8. Santander S, Zubarew T, Santelices L, Argollo P, Cerda J y Bórquez M. Influencia de la familia como factor protector de conductas de riesgo en escolares chilenos. Revista Médica Chile. 2008;136(1):317-324.

9. Di Rico E, Paternain N, y Portillo $\mathrm{N}$. Estudio de características psicosociales relacionadas con riesgo suicida en adolescentes escolarizados de la ciudad de Necochea [Tesis doctoral]. Buenos Aires: Universidad Nacional de Mar del Plata; 2016.

10. Martín M, Pérez R y Riquelme A. Valor diagnóstico de la Escala de Ansiedad y Depresión de Goldberg (EADG) en adultos cubanos. Universitas Psychologica. 2016;15(1):177-192.

11. Gonzales N. Funcionamiento familiar en adolescentes de nivel medio y medio superior [Tesis de maestría]. Nueva León: Universidad Autónoma de Nueva León; 2004.

12. Castilla H, Caycho T, Shimabukuro M, Valdivia A. Percepción del funcionamiento familiar: análisis psicométrico de la Escala APGAR-familiar en adolescentes de Lima. Propósitos y Representaciones. 2014;2(1):49 78.

13. Abambari C, Zhunio D, Romero A, Redrovan J y Atiencia R. La disfuncionalidad familiar como factor asociado a depresión y ansiedad en la población adolescente de Santa Isabel. Revista Médica HJCA 2013;5(1):69-73.

14. Torrel $\mathrm{M}$ y Delgado $\mathrm{M}$. Funcionamiento familiar $y$ depresión en adolescentes de la I.E. Zarumilla-Tumbes 2013. Ciencia y Desarrollo. 2014;17(1):47-53.

15. Lee EO, Park YS, Song M, Lee IS, Park Y Kim HS. Family functioning in the context of chronic illness in women: a Korean study. Int J Nurs Stud. 2002;39(7):705-711.

16. Lopéz M, Barrera M, Cortés J, Guines J. Funcionamiento familiar, creencias e inteligencia emocional en pacientes con trastorno obsesivo-compulsivo y sus familiares. Revista Salud Mental. 2011;34(2):111-120.

17. Mamani B, Quispe C. Depresión y disfunción familiar en estudiantes del cuarto año de secundaria de la Institución Educativa Secundaria Industrial Perú Birf, Juliaca - 2015 [Tesis de pregrado]. Juliaca: Universidad Peruana Unión; 2016.

18. Carrasco M. Factores Clínicos epidemiológicos asociados a depresión en el Perù en el año 2017 [Tesis de pregrado]. Lima: Universidad Ricardo Palma; 2019.

19. Gómez-Maquet Y. Cognición, emoción y sintomatología depresiva en estudiantes escolarizados. Revista Latinoamericana de Psicología. 2007;39(3):435-447.

20. Organización Panamericana de la Salud. Depresión [internet]. 2020 Disponible en: https://www.who.int/es/newsroom/fact-sheets/detail/depression

21. Luengo T y Román J. Estructura familiar y satisfacción parental: Propuestas para la Intervención. Acciones e Investigaciones Sociales. 2006;(1 Ext).

22. Moreno J y Chauta L. Funcionalidad familiar, conductas externalizadas y rendimiento académico en grupo de adolescentes de la ciudad de Bogotá. Psychol Av. Discip. 2012;6(1):155-166.

23. González-Pienda J, Nuñez L, Roces C, Valle A y Bernardo A. Adaptabilidad y cohesión familiar, implicación parental en conductas autorregulatorias, autoconcepto del estudiante y rendimiento académico. Revista Psicothema 2009;15(3):471-477. 